\title{
Effective description of the short-time dynamics in open quantum systems
}

\author{
Matteo A. C. Rossi, ${ }^{1, *}$ Caterina Foti, ${ }^{2,3}$ Alessandro Cuccoli, ${ }^{2,3}$ Jacopo Trapani, ${ }^{1}$ Paola Verrucchi, ${ }^{4,2,3}$ and Matteo G. A. Paris ${ }^{1,5}$ \\ ${ }^{1}$ Quantum Technology Lab, Dipartimento di Fisica, Università degli Studi di Milano, I-20133 Milano, Italy \\ ${ }^{2}$ Dipartimento di Fisica e Astronomia, Università di Firenze, I-50019, Sesto Fiorentino, Florence, Italy \\ ${ }^{3}$ INFN, Sezione di Firenze, I-50019, Sesto Fiorentino, Florence, Italy \\ ${ }^{4}$ ISC-CNR, UOS Dipartimento di Fisica, Università di Firenze, I-50019, Sesto Fiorentino, Florence, Italy \\ ${ }^{5}$ INFN, Sezione di Milano, I-20133, Milano, Italy \\ (Received 3 April 2017; published 20 September 2017)
}

\begin{abstract}
We address the dynamics of a bosonic system coupled to either a bosonic or a magnetic environment and derive a set of sufficient conditions that allow one to describe the dynamics in terms of the effective interaction with a classical fluctuating field. We find that for short interaction times the dynamics of the open system is described by a Gaussian noise map for several different interaction models and independently on the temperature of the environment. In order to go beyond a qualitative understanding of the origin and physical meaning of the above short-time constraint, we take a general viewpoint and, based on an algebraic approach, suggest that any quantum environment can be described by classical fields whenever global symmetries lead to the definition of environmental operators that remain well defined when increasing the size, i.e., the number of dynamical variables, of the environment. In the case of the bosonic environment this statement is exactly demonstrated via a constructive procedure that explicitly shows why a large number of environmental dynamical variables and, necessarily, global symmetries, entail the set of conditions derived in the first part of the work.
\end{abstract}

DOI: 10.1103/PhysRevA.96.032116

\section{INTRODUCTION}

The modeling of any open quantum system (OQS) inherently implies that of its surroundings. However, knowing the quantum structure of the total Hamiltonian, including the details of the couplings between the principal system A and its environment $\Xi$, does not usually suffice to develop a simple and meaningful model of the overall system due to $\Xi$ being made of a very large number $N$ of quantum components, a fact that we will hereafter take as integral to the definition of environment. On the other hand, knowing specific features of $\Xi$ may help in selecting a suitable formalism, and/or some appropriate approximations, so as to devise the most effective strategies for tackling problems that cannot be otherwise studied.

As a matter of fact, the modeling of an effective description of $\Xi$ and of its influence on A usually stems from intuitive and phenomenological arguments [1], or even from an arbitrary choice, rather than a formal derivation. One of the reasons why this is so typical in the study of OQSs is that the large- $N$ theories that have been extensively developed and used in quantum-field theory since the 1970s (comprehensive bibliographies and discussions can be found, for instance, in Refs. [2] and [3]) are not trivially applicable when the large- $N$ system is not isolated but rather coupled with a small, invariably quantum, principal system. Unless one decides that the latter is not "principal" at all and hence can be neglected, several foundational issues arise in this setting, due to the difficult coexistence of quantum and classical formalisms, possibly made worse by the presence of thermal baths or stochastic agents.

Having this issue in mind, here we analyze a specific situation where a principal quantum system $\mathrm{A}$ interacts with an equally quantum environment $\Xi$, which is put into contact

\footnotetext{
*matteo.rossi@unimi.it
}

with a further external system T. If $\Xi$ is macroscopic and T is a thermal bath at high temperature, it may appear intuitive, and naively understood, that A effectively evolves as if it were under the influence of a classical fluctuating field. This statement, however, has the nature of an ansatz as far as it is not formally inferred, and conditions ensuring its validity are not given.

Several OQSs have been investigated indeed to assess whether an effective description is viable, where the effects of the environment are described either with a stochastic Schrödinger equation [4-6] or in terms of the coupling with a classical fluctuating field [7-21]. In the latter case, full equivalence has been shown only for single-qubit dephasing dynamics [9], with an explicit construction of the corresponding classical stochastic process. General arguments valid also for bipartite systems have been discussed [22-24], and the effects of the interaction with a classical field have been investigated in detail [25-32]. Parametric representations have also been used to show that classical variables can emerge in quantum Hamiltonians as environmental degrees of freedom [33-38].

In this work we scrutinize the general idea that the dynamics of a quantum system with a macroscopic environment may be effectively described by a nonautonomous, i.e., timedependent, Hamiltonian acting on the principal system only. In particular, we critically inspect the conditions for the validity of this hypothesis as a tool to understand whether it stems from $\Xi$ being macroscopic, or the temperature being high, or from enforcing some other specific condition.

To this aim, in Secs. II and III we study two specific models that go beyond the pure dephasing and whose analysis will also serve as explicit guidance for the more abstract approach presented in Sec. IV.

In particular, in Sec. II we consider the case where $\mathrm{A}$ is a bosonic mode coupled with an equally bosonic environment, hereafter called $\mathrm{B}$, which is made of $N$ distinguishable modes that do not interact amongst themselves. 
The Hamiltonian reads

$$
\begin{aligned}
H= & v a^{\dagger} a+\sum_{k}^{N}\left(\lambda_{1 k} a^{\dagger}+\lambda_{2 k} a\right) b_{k} \\
& +\sum_{k}^{N}\left(\lambda_{1 k}^{*} a+\lambda_{2 k}^{*} a^{\dagger}\right) b_{k}^{\dagger}+\sum_{k}^{N} \omega_{k} b_{k}^{\dagger} b_{k},
\end{aligned}
$$

where $\left[a, a^{\dagger}\right]=1$ and $\left[b_{k}, b_{k^{\prime}}^{\dagger}\right]=\delta_{k k^{\prime}}$, with $\nu, \omega_{k} \in \mathbb{R}$ and $\lambda_{1 k}, \lambda_{2 k} \in \mathbb{C}, \forall k$. Also, we have set $\hbar=1$, as done throughout this work. Studying the evolution of the reduced density matrix for the principal system, we show that the short-time dynamics defined by Eq. (1), with $\omega_{k} \simeq \omega \forall k$, can be described by an effective Hamiltonian acting on A only, $H_{\mathrm{A}}^{\text {eff }}(\zeta)$, where the functions $\zeta$ embody the remnants of B in the form of classical, possibly fluctuating fields, depending on external parameters such as time and temperature. In what follows we refer to the condition $\omega_{k} \simeq \omega \forall k$ as defining a narrow energy spectrum.

The above model has a sibling that describes the case of a spin environment, hereafter called $\mathrm{S}$, made by $N$ distinguishable spin- $\frac{1}{2}$ particles that do not interact among themselves. Its dynamics is studied in Sec. III, as described by the Hamiltonian

$$
\begin{aligned}
H^{S}= & v a^{\dagger} a+\sum_{i}^{N}\left(g_{1 i} a^{\dagger}+g_{2 i} a\right) \sigma_{i}^{-} \\
& +\sum_{i}^{N}\left(g_{1 i}^{*} a+g_{2 i}^{*} a^{\dagger}\right) \sigma_{i}^{+}+\sum_{i}^{N} f_{i} \sigma_{i}^{z},
\end{aligned}
$$

where $\left[\sigma_{i}^{+}, \sigma_{i^{\prime}}^{-}\right]=2 \delta_{i i^{\prime}} \sigma_{i}^{z},\left[\sigma_{i}^{z}, \sigma_{i^{\prime}}^{ \pm}\right]= \pm \delta_{i i^{\prime}} \sigma_{i}^{ \pm}, f_{i} \in \mathbb{R}$, and $g_{1 i}, g_{2 i} \in \mathbb{C}, \forall i$. Despite that differences with the case of a bosonic environment emerge, essentially due to the specific algebra of the spin operators, the short-time dynamics of this model for $f_{i} \approx f \forall i$ is also found to be properly described by an effective Hamiltonian $H_{\mathrm{A}}^{\text {eff }}(\zeta)$.

Upon inspecting the dynamics of both systems in order to retrace the derivation of the short-time dynamics, we notice that no explicit condition on the value of $N$ is involved. This is somehow surprising, given that $\mathrm{B}$ and $\mathrm{S}$ are named environment insofar as the number $N$ of their quantum components is large, virtually infinite in the case of a macroscopic environment. Therefore, in order to understand whether a relation exists between a large value of $N$ and the assumptions of shorttime and narrow energy spectrum $\omega_{k} \simeq \omega$ used in Secs. II and III, in Sec. IV we take on the model (1) from a more abstract viewpoint. More specifically, we generalize the wellestablished procedures for deriving classical theories as the large- $N$ limit of quantum ones [39] to the case of composite quantum systems, and find that replacing quantum operators by classical fields for $N \rightarrow \infty$ requires that environmental operators stay well defined in such a limit, which, in turn, implies the environment to feature some global symmetry. In particular, we show that the renormalization of the couplings, which is necessary for the $N \rightarrow \infty$ limit to stay physically meaningful, reflects upon the short-time condition previously used. Also, we discuss how a narrow environmental energy spectrum $\omega_{k} \simeq \omega \forall k$ is the key feature that guarantees the existence of a global symmetry in the theory defined by Eq. (1), namely, the symmetry under permutation of different modes.
Overall, collecting our diverse results, we put forward the conjecture that nonautonomous Hamiltonians for closed quantum systems describe the short-time dynamics of interacting models involving at least one macroscopic subsystem. We also comment upon the symmetry properties allowing this subsystem to emerge as a macroscopic one, and the related features of its energy spectrum. Finally, we discuss the role of such symmetry properties in the design of a general procedure for deriving an effective nonautonomous Hamiltonian from an interacting microscopic model.

Section $\mathrm{V}$ closes the paper with some concluding remarks.

\section{BOSONIC ENVIRONMENT}

We consider the Hamiltonian (1), for either (i) $\lambda_{2 k}=0$, with $\lambda_{k} \equiv \lambda_{1 k}$ finite (linear exchange), or (ii) $\lambda_{1 k}=0$, with $\lambda_{k} \equiv \lambda_{2 k}$ finite (parametric hopping), $\forall k$, i.e.,

$$
\begin{aligned}
& H_{1}=v a^{\dagger} a+\sum_{k} \omega_{k} b_{k}^{\dagger} b_{k}+\sum_{k}\left(\lambda_{k}^{*} a b_{k}^{\dagger}+\lambda_{k} a^{\dagger} b_{k}\right), \\
& H_{2}=v a^{\dagger} a+\sum_{k} \omega_{k} b_{k}^{\dagger} b_{k}+\sum_{k}\left(\lambda_{k}^{*} a^{\dagger} b_{k}^{\dagger}+\lambda_{k} a b_{k}\right) .
\end{aligned}
$$

We hereafter use the index $j=1,2$ to refer to the exchange and hopping case, respectively. The Heisenberg equations of motion (EOM) for the mode operators are

$$
\text { Exchange: } \quad \begin{aligned}
& \dot{a}=i\left[H_{1}, a\right]=-i v a-i \sum_{k} \lambda_{k} b_{k}, \\
& \dot{b}_{k}=i\left[H_{1}, b_{k}\right]=-i \omega_{k} b_{k}-i \lambda_{k}^{*} a, \\
& \text { Hopping: } \quad \dot{a}=i\left[H_{2}, a\right]=-i v a-i \sum_{k} \lambda_{k}^{*} b_{k}^{\dagger}, \\
& \dot{b}_{k}^{\dagger}=i\left[H_{2}, b_{k}^{\dagger}\right]=i \omega_{k} b_{k}^{\dagger}+i \lambda_{k} a .
\end{aligned}
$$

If the spectrum of the environment is narrow enough to write $\omega_{k} \simeq \omega \forall k$, the above EOM can be written as

$$
\begin{aligned}
& \text { Exchange: } \dot{a}=-i v a-i \Lambda b, \quad \dot{b}=-i \omega b-i \Lambda a, \\
& \text { Hopping: } \dot{a}=-i v a-i \Lambda b^{\dagger}, \quad \dot{b}^{\dagger}=i \omega b^{\dagger}+i \Lambda a,
\end{aligned}
$$

where the bosonic operator $b$ is defined as

$$
b \equiv \frac{1}{\Lambda} \sum_{k} \lambda_{k} b_{k}, \quad \text { with } \Lambda^{2} \equiv \sum_{k}\left|\lambda_{k}\right|^{2} .
$$

The above Eqs. (7) and (8) are the same EOM that one would obtain starting from the two-mode bosonic Hamiltonians:

$$
\begin{aligned}
& \text { Exchange: } v a^{\dagger} a+\omega b^{\dagger} b+\Lambda\left(a b^{\dagger}+a^{\dagger} b\right), \\
& \text { Hopping: } v a^{\dagger} a+\omega b^{\dagger} b+\Lambda\left(a^{\dagger} b^{\dagger}+a b\right),
\end{aligned}
$$

describing two oscillators, with different frequencies $v$ and $\omega$, exchanging quanta through a linear interaction. Notice, though, that such a direct relation only exists in the case of a narrow spectrum, $\omega_{k} \approx \omega, \forall k$.

Both systems of Eqs. (7) and (8) can be solved by Laplace transform, using the rule $\tilde{a}(s)=s \tilde{a}(s)-a(0)$ to obtain algebraic equations from differential ones. Few calculations 
lead us, after back-transforming and recalling that the index $j=1,2$ refers to the exchange and hopping, respectively, to the solutions

$$
\begin{aligned}
a(t) & =e^{-i H_{j} t} a e^{i H_{j} t}=\left[\mu_{j}(t) a+\pi_{j}(t) \mathcal{B}_{j}\right] e^{-i \omega_{j} t} \\
\mathcal{B}_{j}(t) & =e^{-i H_{j} t} \mathcal{B}_{j} e^{i H_{j} t}=\left[(-)^{j} \pi_{j}^{*}(t) a+\mu_{j}^{*}(t) \mathcal{B}_{j}\right] e^{-i \omega_{j} t}
\end{aligned}
$$

where $\mathcal{B}_{1}=b, \mathcal{B}_{2}=b^{\dagger}$,

$$
\begin{aligned}
& \mu_{j}(t)=\cos \left(\Delta_{j} t\right)-i \frac{\delta_{j}}{\Delta_{j}} \sin \left(\Delta_{j} t\right), \\
& \pi_{j}(t)=-i \frac{\Lambda}{\Delta_{j}} \sin \left(\Delta_{j} t\right),
\end{aligned}
$$

with

$$
\begin{aligned}
\delta_{j} & =\frac{1}{2}\left[v+(-)^{j} \omega\right], \\
\omega_{j} & =\frac{1}{2}\left[v-(-)^{j} \omega\right], \\
\Delta_{j}^{2} & =\left|\delta_{j}^{2}-(-)^{j} \Lambda^{2}\right|,
\end{aligned}
$$

and we have used $\mu_{j}^{*}(t)=\mu_{j}(-t)$. The overall phase factors in the rightmost terms of Eqs. (12) suggest that a natural interaction picture exists, corresponding to frames rotating at frequency $\omega_{j}$. We will use these frames in the following so as to omit those phase factors. Further notice that $\left|\mu_{j}(t)\right|^{2}-$ $(-)^{j}\left|\pi_{j}(t)\right|^{2}=1$, ensuring that $\left[a(t), a^{\dagger}(t)\right]=\left[b(t), b^{\dagger}(t)\right]=$ $1, \forall t$ and also that $\left|\mu_{j}(t)\right|^{2}+(-)^{j} \pi_{j}^{2}(t)=1$, meaning that the evolutions correspond to rotations in the rotating frames.

Our goal is now to obtain an effective Hamiltonian $H_{\mathrm{A}}^{\text {eff }}(\zeta)$, acting on A only, without renouncing the quantum character of its companion B. This means that we can consider nothing but the time dependence of the reduced density matrix for $\mathrm{A}$,

$$
\rho_{\mathrm{A}}(t)=\operatorname{Tr}_{\mathrm{B}}\left[e^{-i H_{j} t} \rho_{\mathrm{A}} \otimes \rho_{\mathrm{B}} e^{i H_{j} t}\right] \equiv \mathcal{E}_{j}\left[\rho_{\mathrm{A}}\right](t),
$$

with the notation $\rho_{\mathrm{X}} \equiv \rho_{\mathrm{X}}(0)$ used hereafter. In particular, as already implied by Eq. (15), we want to derive the explicit form of the dynamical map $\mathcal{E}_{j}\left[\rho_{\mathrm{A}}\right]$ upon assuming that at $t=0$ the system $\mathrm{A}+\mathrm{B}$ is in a factorized state, $\rho_{\mathrm{A}} \otimes \rho_{\mathrm{B}}$. Moreover, we specifically take $B$ initially prepared in the state at thermal equilibrium,

$$
\rho_{\mathrm{B}}=\frac{1}{1+n_{T}}\left(\frac{n_{T}}{1+n_{T}}\right)^{b^{\dagger} b},
$$

where $n_{T}=\left(e^{\omega / T}-1\right)^{-1}$ is the thermal number of photons, and we have set the Boltzmann constant equal to 1 .

After this choice, which implicitly means that B further interacts with a third system $\mathrm{T}$, specifically a thermal bath due to the choice of the state in Eq. (16), we can positively move towards the derivation of the field $\zeta$ entering $H_{\mathrm{A}}^{\text {eff }}$, and of its possible dependence on some external parameter. To this aim we first write the initial state of $A+B$ using the Glauber formula,

$$
\begin{aligned}
\rho_{\mathrm{A}} \otimes \rho_{\mathrm{B}}= & \iint \frac{d^{2} \gamma^{\prime} d^{2} \gamma^{\prime \prime}}{\pi^{2}} \\
& \times \chi\left[\rho_{\mathrm{A}}\right]\left(\gamma^{\prime}\right) \chi\left[\rho_{\mathrm{B}}\right]\left(\gamma^{\prime \prime}\right) D_{a}^{\dagger}\left(\gamma^{\prime}\right) \otimes D_{b}^{\dagger}\left(\gamma^{\prime \prime}\right),
\end{aligned}
$$

where $\chi[\rho](\gamma)=\operatorname{Tr}[\rho D(\gamma)]$ is the characteristic function of the state $\rho$, and $D_{x}(\gamma)=\exp \left\{\gamma x^{\dagger}-\gamma^{*} x\right\}$, with $\left[x, x^{\dagger}\right]=1$, is the bosonic displacement operator. In order to get the argument of the partial trace in Eq. (15), we use Eqs. (12) to write the evolution of the displacement operators entering Eq. (17),

$$
\begin{aligned}
& e^{-i H_{j} t} D_{a}^{\dagger}\left(\gamma^{\prime}\right) \otimes D_{b}^{\dagger}\left(\gamma^{\prime \prime}\right) e^{i H_{j} t} \\
& \quad=D_{a}^{\dagger}\left[\mu_{j}^{*}(t) \gamma^{\prime}+\pi_{j}^{*}(t) \gamma^{\prime \prime}\right] \otimes D_{b}^{\dagger}\left[\pi_{j}^{*}(t) \gamma^{\prime}+\mu_{j}(t) \gamma^{\prime \prime}\right] .
\end{aligned}
$$

We then perform the partial trace using $\operatorname{Tr}[D(\gamma)]=\pi \delta^{(2)}(\gamma)$, so as to get

$$
\begin{aligned}
\mathcal{E}_{j}\left[\rho_{\mathrm{A}}\right](t)= & \int \frac{d^{2} \gamma^{\prime}}{\pi} \chi\left[\rho_{\mathrm{A}}\right]\left(\gamma^{\prime}\right) \chi\left[\rho_{\mathrm{B}}\right] \\
& \times\left(-\frac{\gamma^{\prime} \pi_{j}^{*}(t)}{\mu_{j}(t)}\right) D^{\dagger}\left(\frac{\gamma^{\prime}}{\mu_{j}(t)}\right) \\
= & \int \frac{d^{2} \gamma}{\pi}\left|\mu_{j}(t)\right|^{2} \chi\left[\rho_{\mathrm{A}}\right]\left(\gamma \mu_{j}(t)\right) \\
& \times \chi\left[\rho_{\mathrm{B}}\right]\left(-\gamma \pi_{j}^{*}(t)\right) D^{\dagger}(\gamma),
\end{aligned}
$$

where, in the last step, we made the substitution $\gamma^{\prime} \rightarrow \gamma \mu_{j}(t)$.

Upon expanding the coefficients (13) for $\Delta_{j} t \ll 1$,

$$
\begin{aligned}
\mu_{j}(t) & \simeq 1-i \delta_{j} t+O\left(t^{2}\right), \\
\pi_{j}(t) & \simeq-i \Lambda t+O\left(t^{2}\right), \\
\left|\mu_{j}(t)\right|^{2} & \simeq 1+O\left(t^{2}\right),
\end{aligned}
$$

and using the explicit form of the characteristic function of the thermal state, $\chi\left[\rho_{\mathrm{B}}\right](\gamma)=\exp \left\{-|\gamma|^{2}\left(n_{T}+\frac{1}{2}\right)\right\}$, we finally write

$$
\begin{aligned}
\rho_{\mathrm{A}}(t) & =\mathcal{E}_{j}\left[\rho_{\mathrm{A}}\right](t) \\
& =\int \frac{d^{2} \gamma}{\pi} \chi\left[\rho_{\mathrm{A}}\right](\gamma) e^{-|\gamma|^{2} \sigma^{2}(t)} D^{\dagger}(\gamma),
\end{aligned}
$$

with $\sigma^{2}(t)=\Lambda^{2} t^{2}\left(n_{T}+\frac{1}{2}\right)$.

We now wonder whether the above map is realized by some known unitary evolution involving the interaction with a classical environment only. Indeed, by first noticing that for any state $\varrho$ it is

$$
\chi[\varrho](\gamma) e^{-|\gamma|^{2} \sigma^{2}}=\chi\left[\varrho_{\mathrm{GN}}\right](\gamma),
$$

with

$$
\varrho_{\mathrm{GN}} \equiv \int \frac{d^{2} \alpha}{\pi \sigma^{2}} e^{-\frac{|\alpha|^{2}}{\sigma^{2}}} D(\alpha) \varrho D^{\dagger}(\alpha),
$$

we recognize in Eq. (23) the Kraus decomposition corresponding to a Gaussian noise (GN) channel, namely, a random displacement with Gaussian modulated amplitude [40].

The same map [41,42] describes the evolution of a bosonic system in the presence of a classical fluctuating field, i.e., governed by a nonautonomous Hamiltonian of the form

$$
H_{\text {stoc }}(t)=v a^{\dagger} a+a \zeta^{*}(t) e^{i \omega_{\zeta} t}+a^{\dagger} \zeta(t) e^{-i \omega_{\zeta} t},
$$

where $\zeta(t)$ is a random classical field described by a Gaussian stochastic process $\zeta(t)=\zeta_{x}(t)+i \zeta_{y}(t)$ with zero mean $\left[\zeta_{x}(t)\right]_{\zeta}=\left[\zeta_{y}(t)\right]_{\zeta}=0$ and diagonal structure of the 
autocorrelation function

$$
\begin{aligned}
{\left[\zeta_{x}\left(t_{1}\right) \zeta_{x}\left(t_{2}\right)\right]_{\zeta} } & =\left[\zeta_{y}\left(t_{1}\right) \zeta_{y}\left(t_{2}\right)\right]_{\zeta}=K\left(t_{1}, t_{2}\right), \\
{\left[\zeta_{x}\left(t_{1}\right) \zeta_{y}\left(t_{2}\right)\right]_{\zeta} } & =\left[\zeta_{y}\left(t_{1}\right) \zeta_{x}\left(t_{2}\right)\right]_{\zeta}=0 .
\end{aligned}
$$

The function $\sigma(t)$ in Eq. (22) is in this case

$$
\sigma(t)=\int_{0}^{t} \int_{0}^{t} d t_{1} d t_{2} \cos \left[\delta_{\zeta}\left(t_{1}-t_{2}\right)\right] K\left(t_{1}, t_{2}\right),
$$

where $\delta_{\zeta}=\omega_{\zeta}-v$ is the detuning between the natural frequency $v$ of A and the central frequency $\omega_{\zeta}$ of the classical field $\zeta(t)$. The map (22) may be obtained, for instance, upon considering the classical environment fluctuating according to a Gaussian Ornstein-Uhlenbeck stochastic process [43] characterized by the autocorrelation function

$$
K_{\tau}^{\mathrm{OU}}\left(t_{1}-t_{2}\right)=\frac{G}{2 \tau} e^{-\frac{1}{\tau}\left|t_{1}-t_{2}\right|},
$$

where $\tau$ is the correlation time, and $G$ is the amplitude of the process. In the short-time limit, one easily finds that

$$
\sigma(t)=\frac{G}{2 \tau} t^{2} .
$$

In conclusion, we have shown that, as far as $t \ll\left|\Delta_{j}\right|^{-1}$, the effective Hamiltonian $H_{\mathrm{A}}^{\text {eff }}(\zeta(t))$ equals $H_{\text {stoc }}(t)$, meaning that

$$
H_{\mathrm{A}}^{\mathrm{eff}}(\zeta(t))=v a^{\dagger} a+a \zeta^{*}(t) e^{i \omega_{\zeta} t}+a^{\dagger} \zeta(t) e^{-i \omega_{\zeta} t},
$$

with the field $\zeta(t)$ as from Eqs. (25)-(28), and $G=2 \tau \Lambda^{2}$ $\left(n_{T}+\frac{1}{2}\right)$.

Notice that the dynamical map for A in the short-time limit, Eq. (21), is the same in the exchange and hopping cases. However, due to the $j$ dependence of $\Delta_{j}$, the condition defining the above short-time limit is different in the two cases. In fact, the difference is removed when the number of environmental modes becomes large, and the effective coupling $\Lambda=\sqrt{\sum_{k} \lambda_{k}^{2}}$ increases accordingly, so that

$$
t \ll \frac{1}{\sqrt{\left|(\nu \mp \omega)^{2} \pm \Lambda^{2}\right|}} \underset{\text { large- } N}{\longrightarrow} t \ll \frac{1}{\Lambda},
$$

which establishes a relation between the short-time constraint and some large- $N$ condition that will be further discussed later on.

Overall, we have that the interaction (either exchange or hopping) of an oscillator with a bosonic environment induces a dynamics that is amenable to a description in terms of the interaction with a fluctuating classical field if the following conditions can be, at least approximately, met:

(i) narrow environmental energy spectrum $\left(\omega_{k} \simeq \omega \forall k\right)$,

(ii) short interacting times,

(iii) environment at thermal equilibrium.

It is worth noticing that if conditions (i)-(iii) hold, the above description in terms of classical fields is valid at all temperatures.

\section{MAGNETIC ENVIRONMENT}

We now consider the situation described by the Hamiltonian (2), i.e., that of a bosonic mode A interacting linearly with a magnetic system $\mathrm{S}$, made of $N$ spin- $\frac{1}{2}$ particles, each described by its respective Pauli matrices $\left(\sigma_{i}^{x}, \sigma_{i}^{y}, \sigma_{i}^{z}\right) \equiv \sigma_{i}$. As in Sec. II, we consider both the exchange and the hopping case. Setting (1) $g_{2 i}=0$, with $g_{i} \equiv g_{1 i}$ finite, and (2) $g_{1 i}=0$, with $g_{i} \equiv g_{2 i}$ finite, $\forall i$, from Eq. (2) we get

$$
\begin{aligned}
& H_{1}^{s}=v a^{\dagger} a+\sum_{i} f_{i} \sigma_{i}^{z}+\sum_{i}\left(g_{i}^{*} a \sigma_{i}^{+}+g_{i} a^{\dagger} \sigma_{i}^{-}\right), \\
& H_{2}^{s}=v a^{\dagger} a+\sum_{i} f_{i} \sigma_{i}^{z}+\sum_{i}\left(g_{i}^{*} a^{\dagger} \sigma_{i}^{+}+g_{i} a \sigma_{i}^{-}\right),
\end{aligned}
$$

where the superscript $S$ refers to the magnetic nature of the environment. Setting $f_{i}=f, \forall i$, and further choosing $f>0$, the EOM in the Heisenberg picture are

$$
\begin{aligned}
\text { Exchange: } \quad \dot{a}=i\left[H_{1}^{s}, a\right]=-i v a-i \sum_{i=1}^{N} g_{i} \sigma_{i}^{-}, \\
\dot{\sigma}_{i}^{-}=i\left[H_{1}^{s}, \sigma_{i}^{-}\right]=-i f \sigma_{i}^{-}+i a g_{i}^{*} 2 \sigma_{i}^{z},
\end{aligned}
$$

$$
\text { Hopping: } \begin{aligned}
\quad \dot{a} & =i\left[H_{2}^{S}, a\right]=-i v a-i \sum_{i=1}^{N} g_{i}^{*} \sigma_{i}^{+}, \\
\dot{\sigma}_{i}^{+} & =i\left[H_{2}^{S}, \sigma_{i}^{+}\right]=i f \sigma_{i}^{+}-i a g_{i} 2 \sigma_{i}^{z},
\end{aligned}
$$

where we have related the index of the Hamiltonians $H_{1,2}^{S}$ with the exchange and hopping cases, respectively.

Despite that Eqs. (33) and (34) have the same form as Eqs. (5) and (6) of the bosonic case, they cannot be solved exactly due to the different algebra of the spin operators. However, restricting ourselves to physical situations where the operator $S^{z} \equiv \sum_{i=1}^{N} \sigma_{i}^{z}$ can be replaced by some reasonable expectation value $\left\langle S^{z}\right\rangle \equiv \frac{N}{2}\left\langle\sigma^{z}\right\rangle \equiv-\frac{N}{2} m$ (with $m>0$, due to $f$ being positive), we can rewrite the above EOM in the form

$$
\text { Exchange: } \quad \begin{aligned}
\dot{a} & =-i v a-i \Lambda^{s} \tilde{S}^{-}, \\
\dot{\tilde{S}}^{-} & =-i f \tilde{S}^{-}-i \Lambda^{s} a,
\end{aligned}
$$

$$
\text { Hopping: } \quad \begin{aligned}
\dot{a} & =-i v a-i \Lambda^{s} \tilde{S}^{+}, \\
\dot{\tilde{S}}^{+} & =i f \tilde{S}^{+}+i \Lambda^{s} a,
\end{aligned}
$$

with $g=\sqrt{\sum_{i=1}^{N}\left|g_{i}\right|^{2}}, \Lambda^{s}=g \sqrt{2 m}$, and

$$
\tilde{S}^{+}=\frac{1}{\Lambda^{s}} \sum_{i=1} g_{i} \sigma_{i}^{+}, \quad \tilde{S}^{-}=\left(\tilde{S}^{+}\right)^{\dagger} .
$$

In fact, these equations can be derived from the Hamiltonians

$$
\begin{array}{ll}
\text { Exchange: } & v a^{\dagger} a+f S^{z}+\Lambda^{S}\left(a \tilde{S}^{+}+a^{\dagger} \tilde{S}^{-}\right), \\
\text {Hopping: } & v a^{\dagger} a+f S^{z}+\Lambda^{S}\left(a^{\dagger} \tilde{S}^{+}+a \tilde{S}^{-}\right),
\end{array}
$$

upon further assuming that the commutation relations

$$
\left[\tilde{S}^{+}, \tilde{S}^{-}\right]=-1, \quad\left[S^{z}, \tilde{S}^{+}\right]=\tilde{S}^{+}, \quad\left[S^{z}, \tilde{S}^{-}\right]=-\tilde{S}^{-},
$$

hold, meaning that the spin algebra is simplified into a bosonic one.

Notice that by replacing the total spin operator $\sum_{i} \sigma_{i}^{z}$ with an expectation value $\left\langle S^{z}\right\rangle=\frac{N}{2}\left\langle\sigma^{z}\right\rangle$, we imply that the field $f$ 
selects the same expectation value $\left\langle\sigma^{z}\right\rangle$ for every spin- $1 / 2$, in the spirit of the usual random phase approximation.

Once linearized, the EOM (35) and (36) can be solved as in the bosonic case, to get solutions formally analogous to Eqs. (12) for the operators $a$ and $\tilde{S}_{j}$, with the replacement $\mathcal{B}_{j}(t) \rightarrow \tilde{S}_{j}(t)$ with $\tilde{S}_{1}=\tilde{S}^{-}, \tilde{S}_{2}=\tilde{S}^{+}$, and $\omega \rightarrow f$ in the magnetic expressions corresponding to Eqs. (14).

Whatever follows Eq. (12) in Sec. II can be easily retraced until the choice of the initial environmental state $\rho_{S}$ appears into

$$
\rho_{A}(t)=\operatorname{Tr}_{S}\left[e^{-i H_{j}^{S} t} \rho_{A} \otimes \rho_{S} e^{i H_{j}^{S} t}\right] \equiv \mathcal{E}_{j}^{S}\left(\rho_{A}\right) .
$$

Assuming that $\mathrm{S}$ is initially prepared in a state at thermal equilibrium, we take

$$
\rho_{S}=\frac{1}{1+n_{T}^{s}}\left(\frac{n_{T}^{S}}{1+n_{T}^{s}}\right)^{\tilde{S}^{+} \tilde{S}^{-}},
$$

with $n_{T}^{s} \equiv \frac{N}{2}(1-m)$.

Despite the formal analogy with Eq. (16), it is important to notice that the temperature dependence of $n_{T}^{S}$, and hence that of the dynamical map, is generally different from what we get in the bosonic case, where the thermal number of photons is $n_{T}=\left(\exp \left\{\frac{\omega}{T}\right\}-1\right)^{-1}$. We can, for example, suppose that the magnetic environment thermalizes with the thermal bath so that $\left\langle S^{z}\right\rangle=-\operatorname{sgn}(f) S B_{S}(x)=-\frac{N}{2} \operatorname{sgn}(f) m$, where $S=$ $N / 2$ and $B_{S}(x)=m$ is the Brillouin function

$$
B_{S}(x)=\frac{2 S+1}{2 S} \operatorname{coth}\left(\frac{2 S+1}{2 S} x\right)-\frac{1}{2 S} \operatorname{coth}\left(\frac{x}{2 S}\right),
$$

with $x=S|f| / T$. With this choice, it is $n_{T}^{S} \equiv S\left(1-B_{S}(x)\right)$, and the dependence on $T$ of the bosonic model is only recovered when $T \rightarrow 0$, with $B_{S}(x) \rightarrow 1-e^{-x}$ being the low-temperature limit of Eq. (42). Notice that in order for the above representation to stay meaningful in the large- $S$ limit, temperature must scale as $T \approx S$ so as to guarantee a finite $x$; performing such large- $S$ limit, the Brillouin function turns into the Langevin one, $L(x)=\operatorname{coth}(x)-\frac{1}{x}$, which is indeed the classical limit of Eq. (42).

We observe that the approximations introduced for the spin system are consistent with our aim of finding an effective classical description for the environment: indeed, once the total spin is guaranteed a constant value $S$, a classical-like behavior is expected for a spin system when $S \gg 1[39,44]$, and the bosonic expansion given by the Holstein-Primakoff transformation can be safely truncated at its lowest order $S^{+} \approx$ $b^{\dagger}$ (if $f>0, b^{\dagger}$ being a generic bosonic creation operator) [45].

We can now write the initial state $\rho_{\mathrm{A}} \otimes \rho_{S}$ using the Glauber formula as in Eq. (17), with the spin displacement operator defined as $D_{\tilde{S}}(\gamma)=\exp \left\{\gamma \tilde{S}^{+}-\gamma^{*} \tilde{S}^{-}\right\}$due to the choice $f>0$, and hence $\left\langle\sigma^{z}\right\rangle<0$ (had we taken $f<0$ it would be $\left.D_{\tilde{S}}(\gamma)=\exp \left\{\gamma \tilde{S}^{-}-\gamma^{*} \tilde{S}^{+}\right\}\right)$. Using the solutions of the EOM (35) and (36), one can write the evolution of displacement operators and proceed as done in the previous section up to Eq. (21), thus obtaining that the dynamical map in the magnetic case also corresponds to a Gaussian noise channel. With the additional requirement of a random phase approximation, an effective Hamiltonian of the form of Eq. (29) can hence be written again, allowing us to conclude that the set of conditions sufficient to find an effective classical description is the same as in the bosonic model, the only difference being in the temperature dependence of the standard deviation $\sigma^{2}$, due to the different definition of $n_{T}^{S}$ in the magnetic case.

\section{LARGE- $N$ ENVIRONMENT: DERIVING THE CLASSICAL FIELDS}

In this section we take a more abstract view on the problem of what happens to the principal system A when its environment becomes macroscopic. For the sake of clarity we will specifically refer to the results presented in Secs. II and III and, in particular, to the model (3).

Our aim is to understand whether the emergence of an effective Hamiltonian $H_{\mathrm{A}}^{\text {eff }}(\zeta(t))$ as in Eq. (29) is a general feature of OQSs with macroscopic environments. We also aim at further clarifying the meaning of the conditions (i)-(iii) given at the end of Sec. II, and the reasons why they seem to be utterly necessary in order to obtain an effective Hamiltonian description. Following suggestions from Refs. $[33,35,39,46]$, the main idea is to show that the emergence of $H_{\mathrm{A}}^{\text {eff }}(\zeta)$ is related to the crossover from a quantum to a classical environment, possibly observed when the number of components becomes very large. In fact, were the environment described by a classical theory, its effects on the system would naturally be represented by the classical fields $\zeta$.

Before introducing the general approach we are going to adopt, let us briefly recall some useful notions. A quantum description of a physical system, or quantum theory $Q$ for short, is based on the introduction of (1) a Hilbert space $\mathcal{H}$, (2) a Lie product $[\cdot, \cdot]$ that defines the commutation rules between the operators on $\mathcal{H}$, and (3) a Hamiltonian $H$. Trace class operators on $\mathcal{H}$ that represent physical observables usually make up a vector space; this space, together with the above Lie product, is the Lie algebra $g$ of the theory. The expectation values $\langle O\rangle \equiv\langle\psi|O| \psi\rangle \in \mathbb{R}$ of Hermitian operators are the (only) physical outputs of the theory, i.e., the experimentally accessible properties of the system.

On the other hand, a classical description of a physical system, or classical theory $C$ for short, is defined by (1) a phase space $\mathcal{C},(2)$ a Poisson bracket $\{\cdot, \cdot\}$, and (3) a Hamiltonian $h(\zeta)$, with $\zeta$ representing the set of conjugate variables of the classical phase space $\mathcal{C}$. Real functions $O(\zeta)$ are the (only) physical outputs of the theory, in the same sense as above.

The problem of whether or not a system made by quantum particles can be described by a classical theory has been extensively studied in the last decades of the last century. Different approaches (see, for instance, Sec. VII of Ref. [39] for a thorough discussion) all showed that a large number $N$ of quantum constituents is a necessary condition for a system to admit a classical description, but yet it is not a sufficient one, as confirmed by the experimental observation of macroscopic quantum states. In fact, further conditions must be satisfied that crucially involve symmetry properties of the original quantum theory, and its Lie algebra. Specifically, in Ref. [39] it is demonstrated that the $N \rightarrow \infty$ limit of a quantum theory $Q_{N}$, hereafter indicated by $Q_{N \rightarrow \infty}$, is a classical theory $C$ if $Q_{N}$ exhibits a global symmetry. This latter requirement means that there must exist a group of unitary operators, each acting nontrivially on all of the $N$ constituents, that leave the 
physical observables of the theory invariant (see Ref. [47] for some examples). Indeed, one such symmetry guarantees the existence of a simpler theory $Q_{k}$ (with $k$ a real parameter defined by $N$ ) whose $k \rightarrow 0$ limit, hereafter indicated by $Q_{k \rightarrow 0}$, is physically equivalent to $Q_{N \rightarrow \infty}$, by this meaning that each expectation value that stays finite in the latter limit can be obtained as some expectation value provided by $Q_{k \rightarrow 0}$. On the other hand, $Q_{k \rightarrow 0}$ is also a well-defined classical theory $C$, with phase-space $\mathcal{C}$ and classical Hamiltonian $h(\zeta)$, that therefore provides an effective classical description of the original many-particles quantum system in its macroscopic limit, through the chain $Q_{N \rightarrow \infty}=Q_{k \rightarrow 0}=C$. (See Fig. 3 of Ref. [48] for a graphical depiction of the relation between $Q_{k}, Q_{N}$, and $C$.) Details of the procedure for deriving the above classical theory are given in the Appendix, according to the results presented in Ref. [39] and recently used in the framework of OQSs $[34,48]$. Suffice it here to say that $Q_{N}$ and $Q_{k}$ are related by the fact that their respective Lie algebras, $g_{N}$ and $g_{k}$, are representations of different dimensionality of the same abstract algebra $g$. Let us now get back to our problem, specifically concentrating upon the model described by the Hamiltonian (1). In order to be used in the framework of OQS dynamics, the results mentioned above and the procedure described in the Appendix need to be generalized, as we deal with the quantum theory of a bipartite system where just one of the two constituents, namely, the environment, is intended to become macroscopic. However, due to the linear structure of the interactions entering Eq. (1), the procedure can still be applied as follows.

Keeping in mind that we have to deal with physically meaningful Lie algebras, we first notice that the coupling terms in Eq. (1) can be written as $a\left(a^{\dagger}\right)$ tensor times some sum over $k$ of operators acting on $\mathcal{H}_{\mathrm{B}}$ iff either $\lambda_{1 k}=\lambda_{2 k}$ or $\lambda_{1(2) k}=0$, for all $k$. Taking one or the other of the above conditions true is quite equivalent, as far as the following construction is concerned. For the sake of clarity, and at variance with what was done in Secs. II and III, we specifically choose $\lambda_{2 k}=0$ and set $\lambda_{k} \equiv \lambda_{1 k}$ finite for all $k$, meaning that we explicitly consider the exchange case only. Further taking $\omega_{k}=\omega \forall k$, as done in Secs. II and III, we can define the global operators

$$
E \equiv \frac{1}{N} \sum_{k}^{N} b_{k}^{\dagger} b_{k} \text { and } B \equiv \frac{1}{\sqrt{N \Lambda^{2}}} \sum_{k}^{N} \lambda_{k} b_{k},
$$

with $\Lambda^{2} \equiv \sum_{k}^{N}\left|\lambda_{k}\right|^{2}$ as in Eq. (9), and write the Hamiltonian (1) as

$$
H=v a^{\dagger} a+N\left[\frac{\Lambda}{\sqrt{N}}\left(a^{\dagger} B+a B^{\dagger}\right)+\omega E\right] .
$$

The way $N$ enters Eqs. (43) and (44) is designed to recognize $\frac{1}{N}$ as the parameter to quantify the quantumness of the environment $\mathrm{B}$, and let all the operators, no matter whether acting on $\mathrm{A}, \mathrm{B}$, or $\mathrm{A}+\mathrm{B}$, independent of the number of environmental modes. The operators (43), together with the identity, are easily seen to generate a Heisenberg algebra on $\mathcal{H}_{\mathrm{B}}$, being

$$
\left[B, B^{\dagger}\right]=\frac{1}{N}, \quad[B, E]=\frac{1}{N} B, \quad\left[B^{\dagger}, E\right]=-\frac{1}{N} B^{\dagger}
$$

However, this cannot be regarded as the Lie algebra $g_{N}$ of some environmental theory, due to the presence of noncommuting operators acting on $\mathrm{A}$ in Eq. (44), unless the $N \rightarrow \infty$ limit is taken, as shown below.

Explicitly referring to the example given in Sec. IV of Ref. [39] and the strategy described in the Appendix, we introduce the set of anti-Hermitian operators

$$
\left\{L(\epsilon, \beta) \equiv i N\left(\epsilon E+\beta^{*} B+\beta B^{\dagger}\right)\right\},
$$

where $\beta \in \mathbb{C}$, with $|\beta| \propto \frac{1}{\sqrt{N}}$, while the coefficients $\in \in \mathbb{R}$ do not depend on $N$. In the large- $N$ limit, where terms which are bilinear in $\beta$ and $\beta^{*}$ can be neglected due to their dependence on $N$, it is $\left[L_{1}, L_{2}\right]=L_{3}$, with $L_{i} \equiv L_{i}\left(\epsilon_{i}, \beta_{i}\right), \beta_{3}=i\left(\epsilon_{1} \beta_{2}-\right.$ $\epsilon_{2} \beta_{1}$ ), and $\epsilon_{3}=0$, meaning that the set (46) is a Lie algebra. This is indeed the algebra $g_{N}$, whose recognition represents the first step towards the large- $N$ limit of the quantum theory that describes B. It is easily checked that a possible representation $g_{k}$, of the same abstract algebra represented by $g_{N}$, is given by the $2 \times 2$ matrices

$$
\left\{\ell(\epsilon, \beta) \equiv i\left(\begin{array}{cc}
0 & \beta^{*} \\
0 & \epsilon
\end{array}\right)\right\},
$$

being $\left[\ell_{1}, \ell_{2}\right]=\ell_{3}$, with $\ell_{i} \equiv \ell_{i}\left(\epsilon_{i}, \beta_{i}\right)$, and $\beta_{3}, \epsilon_{3}$ as above. We underline that the choice of a representation $g_{k}$ that contains only either $\beta$ or $\beta^{*}$ is the simplest way to make the presence of noncommuting operators on $\mathcal{H}_{\mathrm{A}}$ in the Hamiltonian (44) harmless as far as the following construction is concerned.

The matrices $\ell(\epsilon, \beta)$ allow us to write

$$
\left[L,\left(\begin{array}{c}
1 \\
B
\end{array}\right)\right] \equiv\left(\begin{array}{c}
{[L, 1]} \\
{[L, B]}
\end{array}\right)=\left(\begin{array}{c}
0 \\
-i\left(\epsilon B+\beta^{*}\right)
\end{array}\right)
$$

as

$$
\left[L,\left(\begin{array}{l}
1 \\
B
\end{array}\right)\right]=\ell^{\dagger}\left(\begin{array}{l}
1 \\
B
\end{array}\right),
$$

with $\ell^{\dagger} \equiv\left(\ell^{*}\right)^{t}$, and, quite equivalently,

$$
\left[L,\left(1 \quad B^{\dagger}\right)\right]=\left(\begin{array}{ll}
1 & B^{\dagger}
\end{array}\right) \ell .
$$

Let us now consider the unitary operators

$$
U(\epsilon, \beta) \equiv \exp \{L(\epsilon, \beta)\}:
$$

given that, for any pair of operators $O$ and $P$, it holds that

$$
e^{-P} O e^{P}=\sum_{n} \frac{(-1)^{n}}{n !}[P,[P,[\ldots[P, O] \ldots]]],
$$

from Eqs. (49) and (50) it follows

$$
U^{-1}\left(\begin{array}{l}
1 \\
B
\end{array}\right) U=u(\phi, \zeta)\left(\begin{array}{l}
1 \\
B
\end{array}\right)
$$

and

$$
U^{-1}\left(1 \quad B^{\dagger}\right) U=\left(1 \quad B^{\dagger}\right) u^{\dagger}(\phi, \zeta),
$$

with

$$
u(\phi, \zeta) \equiv\left(\begin{array}{ll}
1 & 0 \\
\zeta & \phi
\end{array}\right)
$$

where

$$
\phi=e^{i \epsilon} \text { and } \zeta=\frac{\beta}{\epsilon}\left(e^{i \epsilon}-1\right)
$$


are obtained by explicitly summing the series in Eq. (52).

The fact that the set (46) is a Lie algebra in the large- $N$ limit reflects upon the unitary operators $U(\phi, \zeta)$, in that they form a group in the same limit. In fact, this is just the Lie group corresponding to $g_{k}$, sometimes dubbed the dynamical [49] or coherence [50] group, that defines, together with the arbitrary choice of a reference state $|0\rangle \in \mathcal{H}_{\mathrm{B}}$, the generalized coherent states (GCS) $|u(\phi, \zeta)\rangle \equiv U(\phi, \zeta)|0\rangle$ for the theory $Q_{k}$. The reason why these states are so relevant, as further commented upon in the Appendix, is that the operators $B$ and $E$ are demonstrated [39] to transform into $B(u) \equiv\langle u|B| u\rangle / N$ and $E(u) \equiv\langle u|E| u\rangle / N$, respectively, as $N$ goes to infinity. Therefore, in order to find the large- $N$ limit of the Hamiltonian (44), we now only need to evaluate $B(u)$ and $E(u)$, even without knowing the explicit form of the GCS, to obtain $H_{N}^{\text {eff }}(\zeta)$ from

$$
\begin{aligned}
& \underset{N \rightarrow \infty}{H} v a^{\dagger} a+N\left[\frac{\Lambda}{\sqrt{N}}\left(a^{\dagger} B(u)+a B^{*}(u)\right)+\omega E(u)\right] \\
& \quad \equiv H_{N}^{\mathrm{eff}}(\zeta)
\end{aligned}
$$

where the relation between $|u\rangle$ and $\zeta$ is made explicit below. To proceed accordingly, we choose the reference state for the GCS: $|0\rangle=\Pi_{k}|0\rangle_{k}$, with $|0\rangle_{k}$ such that $b_{k}|0\rangle_{k}=0$. This implies, given the separable structure of the operators $U(\phi, \zeta)$, that the states $|u\rangle$ are tensor products of singlemode pure states. As a consequence, it is $\left\langle u\left|B B^{\dagger}\right| u\right\rangle=$ $\left\langle u\left|\sum_{k^{\prime} k} b_{k^{\prime}} b_{k}^{\dagger}\right| u\right\rangle=\left\langle u\left|\sum_{k} b_{k} b_{k}^{\dagger}\right| u\right\rangle=N E(u)$, which allows us to determine $B(u)$ and $E(u)$ via

$$
\left\langle u\left|\left(\begin{array}{c}
1 \\
B
\end{array}\right) \otimes\left(\begin{array}{ll}
1 & B^{\dagger}
\end{array}\right)\right| u\right\rangle=N\left(\begin{array}{cc}
1 & B^{*}(u) \\
B(u) & E(u)
\end{array}\right),
$$

and finally obtain, by Eqs. (53) and (54) and again neglecting terms bilinear in $\beta$ and $\beta^{*}$,

$$
\begin{aligned}
& \left\langle 0\left|u(\phi, \zeta)\left(\begin{array}{l}
1 \\
B
\end{array}\right) \otimes\left(1 \quad B^{\dagger}\right) u^{\dagger}(\phi, \zeta)\right| 0\right\rangle \\
& =\left\langle 0\left|\left(\begin{array}{cc}
1 & \zeta^{*}+\phi^{*} B^{\dagger} \\
\zeta+\phi B & \zeta \zeta^{*}+\zeta \phi^{*} B^{\dagger}+\zeta^{*} \phi B+\phi \phi^{*} B B^{\dagger}
\end{array}\right)\right| 0\right\rangle \\
& =\left(\begin{array}{cc}
1 & \zeta^{*} \\
\zeta & 1
\end{array}\right)
\end{aligned}
$$

i.e., $E(u)=1 / N$ and $B(u)=\zeta / N$.

The above result implies that the original Hamiltonian (44) formally transforms, according to Eq. (57), as

$$
H \underset{N \rightarrow \infty}{\longrightarrow} H_{\mathrm{A}}^{\mathrm{eff}}(\zeta)=\left(v a^{\dagger} a+\omega\right)+\zeta^{*} a+\zeta a^{\dagger},
$$

where we have rescaled $\zeta \rightarrow \zeta \Lambda / \sqrt{N}$, and $\left(\zeta, \zeta^{*}\right) \in \mathbb{R}^{2}$ is generally proved [39] to be any point of a classical phase-space $\mathcal{M}_{\mathrm{B}}$ with canonical variables $q \equiv\left(\zeta+\zeta^{*}\right) / 2$ and $p \equiv(\zeta-$ $\left.\zeta^{*}\right) /(2 i)$. Notice that $|\zeta| \propto \Lambda / \sqrt{N}$, which is independent of $N$ by definition.

Once Eq. (60) is obtained, we can maintain with confidence that the Hamiltonian (1), originally acting on $\mathrm{A}+\mathrm{B}$, formally transforms, as $N \rightarrow \infty$, into one that exclusively acts on $\mathrm{A}$. However, the presence of the classical field $\zeta$ is the remnant of the underlying quantum interaction between $\mathrm{A}$ and the huge number of elementary constituents of $\mathrm{B}$, namely, the bosonic modes $\left\{b_{k}\right\}_{k=1}^{N}$. To this respect, notice that the Hilbert space
$\mathcal{H}_{\mathrm{B}}=\otimes_{k} \mathcal{H}_{\mathrm{b}_{k}}$ is replaced by a two-dimensional classical phase space $\mathcal{M}_{\mathrm{B}}$, implying an impressive reduction of dynamical variables. This reduction is the most striking consequence of the global symmetry that the quantum theory for B must exhibit in order to flow into a well-defined classical theory when B is macroscopic. In our case, although we did not explicitly used it, the symmetry is that under permutation of the bosonic modes $b_{k}$, and that is why we have set $\omega_{k}=\omega \forall k$. In fact, one can easily check that this is an essential condition for the very same definition of global operators obeying commutation rules of the form (45), which in their turn are necessary to proceed to the definition of the Lie algebra, and all the rest.

At this point, we notice that $\omega_{k}=\omega \forall k$ is just the "narrow environmental energy-spectrum condition" (i), discussed at the end of Sec. II. In fact, it is immediately striking that the effective Hamiltonian in Eq. (60) has the same structure of that in Eq. (29), given that the latter refers to an interaction picture that hides the environmental frequency $\omega$. On the other hand, it is somehow puzzling that time does not enter the above construction, which leave us clueless, so far, concerning the relation $\zeta \rightarrow \zeta(t) e^{-i \omega_{\zeta} t}$.

Looking for the possible origin of a time dependence in the classical field $\zeta$, we reckon that the results of this section imply the following: Suppose there exists another macroscopic system $\mathrm{T}$ that is not coupled with $\mathrm{A}$, and interacts with $\mathrm{B}$ in such a way that the above global symmetry is preserved. The presence of $\mathrm{T}$ manifests itself in terms of some parameter $\tau$ (think about time and/or temperature, for instance) upon which $\zeta$ depends, according to the rule $\zeta=\zeta(\tau)$ provided by the classical theory describing $\mathrm{B}+\mathrm{T}$. This dependence can be safely imported into the effective description of A via $\zeta \rightarrow$ $\zeta(\tau)$ in $H_{\mathrm{A}}^{\text {eff }}(\zeta)$, Eq. (60), as far as the direct interaction between $\mathrm{A}$ and $\mathrm{T}$ can be neglected, at least on the time scales one is interested in.

Finally, we notice that the detuning $v-\omega$ does not play any role in this section, which brings us back to Eq. (30) and the possible relation between the large- $N$ condition here enforced and the short-time approximation previously adopted.

\section{CONCLUSIONS}

In this paper, we have addressed the dynamics of a bosonic system coupled to either a bosonic or a magnetic environment. In particular, we have discussed the conditions under which the dynamics of the system may be described in terms of the effective interaction with a classical fluctuating field.

Our results show that for both kinds of environments an effective, time-dependent Hamiltonian description may be obtained for short interaction times and environments with a narrow energy spectrum at thermal equilibrium. The corresponding dynamics is described by a Gaussian noise channel independently of the kind of environment, their magnetic or bosonic nature entering only the form of the noise variance. As far as the energy spectrum is narrow, this effective description is valid at all temperatures and independently of the nature of the interaction between the system and its environment.

Moreover, exploiting a general treatment based on the large$N$ limit of the environment, we have clarified the origin and the meaning of the narrow environmental spectrum and short-time 
conditions. In fact, we find that $\omega_{k} \simeq \omega \forall k$ is needed for a global symmetry to emerge and characterize the environment, which is a necessary ingredient for the environment to be described by a small number of macroscopic variables. On the other hand, the large energy scale implied by whatever coupling with a macroscopic environment limits any effective description to short times only.

Overall, our results indicate that quantum environments may be described by classical fields whenever global symmetries allow one to define environmental operators that remain well defined when increasing the spatial size of the environment. This is a quite general criterion that may serve as a guideline for further analysis, e.g., for fermionic principal systems and/or hybrid environments.

\section{ACKNOWLEDGMENTS}

This work has been supported by UniMI through H2020 Transition Grant No. 15-6-3008000-625, and by EU through the Collaborative Projects QuProCS (Grant Agreement No. 641277). P.V. has worked in the framework of the Convenzione Operativa between the Institute for Complex Systems of the Italian National Research Council (CNR) and the Department of Physics and Astronomy of the University of Florence.

\section{APPENDIX}

Consider a system made by $N$ elements which is described by a quantum theory $Q_{N}$ that features a global symmetry, as defined in Sec. IV. (We will equip quantities with the index $N$ to indicate their being relative to this $Q_{N}$ theory.) The procedure described in Ref. [39] for deriving the classical theory that formally represents $Q_{N \rightarrow \infty}$ can be summarized as follows. The first step is that of identifying $g_{N}$, exploiting the knowledge of the Hilbert space $\mathcal{H}=\otimes_{i}^{N} \mathcal{H}_{i}$, the Lie product, and the Hamiltonian $H_{N}$. As the Hamiltonian $H_{N}$ represents a physical observable, an effective strategy to identify $g_{N}$ is that of writing $H_{N}$ as a linear combination of operators and see if they belong to some minimal set that generates a representation $g_{N}$ of some abstract Lie algebra $g$. The second step of the procedure is that of finding an irreducible representation $g_{k}$ of $g_{N}$, which stands as the Lie algebra for $Q_{k}$. (Notice that this most often implies that an explicit expression for $H_{k}$ does also become available.) Here is where the existence of a global symmetry emerges as a necessary ingredient, as it guarantees that the dimensionality of the representation $g_{k}$ be significantly smaller than that of $g_{N}$. In fact, the way $g_{k}$ can be most often identified is writing the original Hamiltonian as a linear combination of some global (i.e., acting nontrivially upon each subsystem) operators that are invariant under the symmetry operations, and generate a representation of the same abstract algebra $g$ which is also represented by $g_{N}$. In the third step, generalized coherent states (GCSs) for $Q_{k}$ come into play. These are defined, according to the approach either of Gilmore et al. [49] or, quite equivalently, of Perelomov [50], starting from the dynamical group of the theory, which is nothing but the Lie group associated to $g_{k}$ by the usual Lie correspondence [51], and is therefore provided by the above second step. GCS for $Q_{k}$, hereafter indicated by $|u\rangle \in \mathcal{H}_{k}$, enter the procedure due to their being [49] in one-to-one correspondence with points $u$ on a manifold $\mathcal{M}_{k}$, whose cotangent bundle is a classical phase-space $\mathcal{C}$. In other terms, each GCS $|u\rangle$ of the theory $Q_{k}$ defines a point $u \in \mathcal{M}_{k}$ and a set of conjugate variables $\zeta \in \mathcal{C}$. In fact, it is demonstrated [39] that $Q_{k \rightarrow 0}$ is a classical theory $C$, with phase space the above cotangent bundle $\mathcal{C}$, and Hamiltonian $h(\zeta)=\left\langle u\left|H_{k}\right| u\right\rangle / N$. The last step of the procedure is that of deriving, possibly without knowing the explicit form of the GCS, the expectation values $\left\langle u\left|H_{k}\right| u\right\rangle$, and finally obtain the effective classical Hamiltonian describing the original quantum system in the $N \rightarrow \infty$ limit. The role of the parameters $N$ and $k$, which has been here understood for the sake of a lighter narration, becomes evident when explicitly employing the procedure, as in Sec. IV, where it is $k=1 / N$.
[1] R. Alicki and K. Lendi, Quantum Dynamical Semigroups and Applications, Lecture Notes in Physics Vol. 717 (SpringerVerlag, Berlin, 2007).

[2] E. Brézin and S. R. Wadia, The Large $N$ Expansion in Quantum Field Theory and Statistical Physics (World Scientific, Singapore, 1993).

[3] A. Auerbach, Interacting Electrons and Quantum Magnetism (Springer, New York, 1994).

[4] W. T. Strunz, L. Diósi, and N. Gisin, Phys. Rev. Lett. 82, 1801 (1999).

[5] J. T. Stockburger and H. Grabert, Phys. Rev. Lett. 88, 170407 (2002).

[6] D. Lacroix, Phys. Rev. E 77, 041126 (2008).

[7] J. Helm and W. T. Strunz, Phys. Rev. A 80, 042108 (2009).

[8] J. Helm, W. T. Strunz, S. Rietzler, and L. E. Würflinger, Phys. Rev. A 83, 042103 (2011).

[9] D. Crow and R. Joynt, Phys. Rev. A 89, 042123 (2014).

[10] W. M. Witzel, K. Young, and S. D. Sarma, Phys. Rev. B 90, 115431 (2014).
[11] G. M. Palma, K.-A. Suominen, and A. K. Ekert, Proc. R. Soc. London, Ser. A 452, 567 (1996).

[12] E. Paladino, L. Faoro, G. Falci, and R. Fazio, Phys. Rev. Lett. 88, 228304 (2002).

[13] Ł. Cywiński, R. M. Lutchyn, C. P. Nave, and S. D. Sarma, Phys. Rev. B 77, 174509 (2008).

[14] H. J. Wold, H. Brox, Y. M. Galperin, and J. Bergli, Phys. Rev. B 86, 205404 (2012).

[15] J.-T. Hung, Ł. Cywiński, X. Hu, and S. D. Sarma, Phys. Rev. B 88, 085314 (2013).

[16] E. Paladino, M. Y. Galperin, G. Falci, and L. B. Altshuler, Rev. Mod. Phys. 86, 361 (2014).

[17] R. Lo Franco and G. Compagno, in Lectures on General Quantum Correlations and Their Applications, edited by F. Fanchini, D. de O. Soares Pinto, and G. Adesso (Springer International Publishing, Cham, 2017), pp. 367-391.

[18] A. Orieux, A. D’Arrigo, G. Ferranti, R. Lo Franco, G. Benenti, E. Paladino, G. Falci, F. Sciarrino, and P. Mataloni, Sci. Rep. 5, 8575 (2015). 
[19] R. Lo Franco, B. Bellomo, E. Andersson, and G. Compagno, Phys. Rev. A 85, 032318 (2012).

[20] B. Leggio, R. Lo Franco, D. O. Soares-Pinto, P. Horodecki, and G. Compagno, Phys. Rev. A 92, 032311 (2015).

[21] J.-S. Xu, K. Sun, C.-F. Li, X.-Y. Xu, G.-C. Guo, E. Andersson, R. Lo Franco, and G. Compagno, Nat. Commun. 4, 2851 (2013).

[22] C. Benedetti and M. G. A. Paris, Int. J. Quantum Inform. 12, 1461004 (2014).

[23] J. Shao, J. Chem. Phys. 120, 5053 (2004).

[24] M. A. C. Rossi, C. Benedetti, and M. G. A. Paris, Int. J. Quantum Inf. 12, 1560003 (2014).

[25] J. Trapani and M. G. A. Paris, Phys. Rev. A 93, 042119 (2016).

[26] Y. M. Galperin, B. L. Altshuler, J. Bergli, and D. V. Shantsev, Phys. Rev. Lett. 96, 097009 (2006).

[27] C. Benedetti, F. Buscemi, P. Bordone, and M. G. A. Paris, Phys. Rev. A 87, 052328 (2013).

[28] T. Yu and J. H. Eberly, Opt. Commun. 283, 676 (2010).

[29] C. Benedetti, M. G. A. Paris, and S. Maniscalco, Phys. Rev. A 89, 012114 (2014).

[30] M. A. C. Rossi and M. G. A. Paris, J. Chem. Phys. 144, 024113 (2016).

[31] C. Benedetti and M. G. A. Paris, Phys. Lett. A 378, 2495 (2014).

[32] M. A. C. Rossi and M. G. A. Paris, Phys. Rev. A 92, 010302 (2015).

[33] D. Calvani, A. Cuccoli, N. Gidopoulos, and P. Verrucchi, Proc. Natl. Acad. Sci. USA 110, 6748 (2013).

[34] P. Liuzzo-Scorpo, A. Cuccoli, and P. Verrucchi, Int. J. Theor. Phys. 54, 4356 (2015).

[35] C. Foti, A. Cuccoli, and P. Verrucchi, Phys. Rev. A 94, 062127 (2016).

[36] W. T. Strunz, Open Sys. Info. Dyn. 12, 65 (2005).

[37] A. Pernice, J. Helm, and W. T. Strunz, J. Phys. B: At., Mol. Opt. Phys. 45, 154005 (2012).
[38] A. Pernice and W. T. Strunz, Phys. Rev. A 84, 062121 (2011).

[39] L. G. Yaffe, Rev. Mod. Phys. 54, 407 (1982).

[40] To prove the equivalence in Eq. (22), start from $\operatorname{Tr}\left[\rho_{\mathrm{GN}}+\right.$ $D(\gamma)$ ], insert the definition of $\rho_{\mathrm{GN}}$, use the composition rule $D^{\dagger}(\alpha) D(\gamma) D(\alpha)=D(\gamma) e^{\alpha^{*} \gamma-\gamma^{*} \alpha}$, and perform the resulting Fourier transform.

[41] R. R. Puri, Mathematical Methods of Quantum Optics (SpringerVerlag, Berlin, 2001).

[42] J. Trapani, M. Bina, S. Maniscalco, and M. G. A. Paris, Phys. Rev. A 91, 022113 (2015).

[43] G. E. Uhlenbeck and L. S. Ornstein, Phys. Rev. 36, 823 (1930).

[44] E. H. Lieb, Commun. Math. Phys. 31, 327 (1973).

[45] J. M. Radcliffe, J. Phys. A 4, 313 (1971).

[46] A. Cuccoli, A. Taiti, R. Vaia, and P. Verrucchi, Phys. Rev. B 76, 064405 (2007).

[47] If the theory describes $N$ spin-1/2 particles interacting via an isotropic Heisenberg-like magnetic exchange, one such symmetry can be that defined by operators that rotate the spin of each particle of the same angle. Take instead $N$ particles whose interaction depends only on their distance; the symmetry might be that defined by the same spatial translation of each particle. As for noninteracting, identical, but yet distinguishable particles, a possible global symmetry is that defined by the permutation operators.

[48] P. Liuzzo-Scorpo, A. Cuccoli, and P. Verrucchi, Europhys. Lett. 111, 40008 (2015).

[49] W.-M. Zhang, D. H. Feng, and R. Gilmore, Rev. Mod. Phys. 62, 867 (1990).

[50] A. Perelomov, Commun. Math. Phys. 26, 222 (1972).

[51] R. Gilmore, Lie Groups, Lie Algebras, and Some of Their Applications, Dover Books on Mathematics (Dover Publications, New York, 2012). 